\title{
Critical Junction: Nonlinear Dynamics, Swarm Intelligence and Cancer Research
}

\author{
Simon Rosenfeld* \\ National Cancer Institute, Division of Cancer Prevention, USA
}

\begin{abstract}
Complex biological systems manifest a large variety of emergent phenomena among which prominent roles belong to self-organization and swarm intelligence. Despite astoundingly wide repertoire of observed forms, there are comparatively simple rules governing evolution of large systems towards self-organization, in general, and towards swarm intelligence, in particular. In this work, an attempt is made to outline general guiding principles in exploration of a wide range of seemingly dissimilar phenomena observed in large communities of individuals devoid of any personal intelligence and interacting with each other through simple stimulus-response rules. Mathematically, these guiding principles are well captured by the Global Consensus Theorem (GCT) allowing for unified approach to such diverse systems as biological networks, communities of social insects, robotic communities, microbial communities, communities of somatic cells, to social networks, and to many other systems. The GCT provides a conceptual basis for understanding the emergent phenomena of self-organization occurring in large communities without involvement of a supervisory authority, without system-wide informational infrastructure, and without mapping of general plan of action onto cognitive/ behavioral faculties of its individual members. Cancer onset and proliferation serves as an important example of application of these conceptual approaches. A growing body of evidence confirms the premise that disruption of quorum sensing, an important aspect of swarm intelligence, plays a key role in carcinogenesis. Other aspects of swarm intelligence, such as collective memory, adaptivity (a form of learning from experience) and ability for self-repair are the key for understanding biological robustness and acquired chemoresistance. Yet another aspects of swarm intelligence, such as division of labor and competitive differentiation, may be helpful in understanding of cancer compartmentalization and tumor heterogeneity.
\end{abstract}

\section{Introduction}

Swarm intelligence of social insects and microbial colonies vividly demonstrates how far the evolution may progress having at its disposal only simple rules of interaction between unsophisticated individuals. The Lotka-Volterra (LVS) family of mathematical models, being among the first models capable of describing the very complex systems with very simple rules of interactions, demonstrates how complex may be the behaviors of even a simple food web consisting of only one predator and one prey. The repertoire of behaviors of multispecies populations is virtually unlimited. In particular, it has been shown that swarm intelligence may originate from rather mundane reasons rooted in simple rules of interactions between these entities. The goal of this paper is to provide a brief overview of properties of the multidimensional nonlinear dynamical systems which have a potential of producing the phenomenon of self-organized behavior and manifesting themselves as swarm intelligence.

\section{Swarm intelligence, definitions and manifestations}

By definition, swarm intelligence is the organized behavior of large communities without global organizer and without mapping the global behavior onto the cognitive/behavioral abilities of the individual members of the community [1]. It should be emphasized that what is called here communities are not necessarily the communities of living entities like bee hives or ant hills or microbial colonies. Moreover, complexity of collective behavior of the community as a whole does not require its individual members to have any extensive analytical tools or even memory on their own. The key prerequisite for the possibility of community-wide self-organization is that individual members may interact following the stimulus-response rules. Large-scale community- wide behaviors and self-organized modalities are completely determined by these low-level local interactions. There are a number of closely related but distinctly different aspects of swarm intelligence. These are collective memory, adaptivity, division of labor, cooperation, sensing of environment (a.k.a., stigmergy) and quorum sensing. All these aspects are the emergent properties resulting from local memberto-member interactions without a general plan of action, without a supervisory authority, and without a system-wide information infrastructure. From the mathematical standpoint, a large system of locally interacting units is a dynamic network governed by the laws of nonlinear dynamics. The following question, therefore, is in order: what exactly are the laws of local interactions leading to emergence of complex behaviors which are referred to as swarm intelligence?

\section{Mechanistic origins of self-organization and swarm intelligence}

A comparatively simple, and abundantly well studied, example of the system manifesting the property of swarm intelligence is neural network $(\mathrm{NN})[2,3]$. NN functionality originates from and closely mimics the neuronal networks constituting the nervous systems of higher organisms. Among the analytical tools collectively known as

Correspondence to: Simon Rosenfeld, National Cancer Institute, Division of Cancer Prevention, 9609 Medical Center Drive, rm 5E640, Rockville, MD 20850, USA, E-mail: sr212a@nih.gov

Key words: global consensus theorem, swarm intelligence, biomolecular networks, carcinogenesis

Received: April 13, 2015; Accepted: May 16, 2015; Published: May 20, 2015 
artificial intelligence, NNs retain the leading positions in a variety of computational tasks; among them are pattern recognition and classification, short- and long-term storage of information, prediction and decision making, optimization, and other. Due to the fundamental property of being universal approximators, the NNs are capable, in principle, of representing any nonlinear dynamical system. Such systems may possess a number of asymptotically stable attractors. This means that starting with a large variety of initial conditions belonging to a certain basin of attraction the system may evolve towards one of the several well-defined stable manifolds. This process is in fact nothing other than classification of initial states which occurs in the system without any organizational force or supervisory authority.

The Lotka-Volterra Systems (LVS) is a large class of dynamical systems described by the ordinary differential equation with quadratic nonlinearities [4]. Originally inspired by ecology and population dynamics, the LVS theory largely retains their flavors and terminology. In particular, independent variables are assumed to be the population levels of corresponding species, the coefficients describe the rates of reproduction and extinction. Interactions between the species may be mutualistic (cooperative) or antagonistic (competitive). This terminology evokes dramatic visions of struggle for survival, either individually or collectively, so frequently observed in the world of living entities. However, from the mathematical standpoint, there is nothing dramatic in the LVS dynamics: all the systems describable by LVS, whether belonging to biological, or physical, or technological, or social, or financial realms, will have similar dynamical behaviors and analogous emergent properties. Due to this reason, and in order to avoid direct ecological connotations, the variables in LVS are often called quasi-species thus emphasizing that the actual nature of these species is of secondary importance.

A fundamental question pertaining to competitive LVS is the question of dynamic stability. In the context of population dynamics, stability means that, despite the fact that all the species are struggling with each other, they may nevertheless come to some sort of peaceful coexistence or consensus regarding the distribution of limited resources. Since nothing except the pair-wise interactions is included in LVS dynamics, this consensus cannot be a result of collective decision making or planning. The challenge and fundamental importance of the question of stability have been articulated by S. Grossberg: "The following problem, in one form or another, has intrigued philosophers and scientists for hundred of years: How do arbitrarily many individuals, populations, or states, each obeying unique and personal laws, ever succeed in harmoniously interacting with each other to form some sort of stable society, or collective mode of behavior? Otherwise expressed, if each individual obeys complex laws, and is ignorant of other individuals except via locally received signals, how is social chaos averted? How can local ignorance and global order, or consensus, be reconciled? ...What design constrains must be imposed on a system of competing populations in order that it be able to generate a global limiting pattern, or decision, in response to arbitrary initial data?... How complicated can a system be and still generate order? [5]”.

The questions outlined above have been successfully resolved within a wide class of competitive nonlinear dynamical systems, with NNs and LVS being their particular cases. In order to avoid cumbersome mathematical notation and explicit definitions within this paper we will call these system G-systems. The fundamental Global Consensus Theorem (GCT), proved by S. Grossberg in a series of publications [5-8] claims that within the class of G-systems the tendency to selforganization is rooted in fairly simple nature of things: any complex system whose unstoppable growth is inhibited by progressively dwindling resources will end up with some sort of self-structuring and consensus regarding the distribution of resources. Generality and simplicity of the G-systems dynamics guarantees its applicability to very wide class of natural, technological and societal phenomena. Transition from the dominance of one quasi-species to another may appear as a struggle for survival, and it is indeed an existential struggle in the predator-prey food chains. Although the metaphor of struggle for survival is widely used beyond the world of living entities, it is obvious from the GCT that the reasons for competitive dynamics leading to consensus may be much simpler and may have nothing to do with personal motivation of a living entity to survive. In this context, it is not out of place to recall that the co-founder of LVS, Alfred Lotka, pointed out that natural selection should be approached more like a physical principle subject to treatment by the methods of statistical mechanics, rather than as struggle of living creatures motivated by the desire to survive [9].

The GCT provides a deep insight into the seemingly miraculous property of complex hierarchical systems to be self-organized at each level without supervisory authority, without informational infrastructure, without necessity for its units to have understanding of the process as a whole, and without invoking the metaphor of struggle for survival. The GST also provides the clues on how such complex emergent phenomenon as swarm intelligence may appear in the systems consisting of only unsophisticated individuals devoid of any personal intelligence and interacting with each other only through simple pair-wise stimulus-response rules.

\section{Swarm intelligence in G-systems}

\section{Chemical networks}

Perhaps the simplest G-system fully satisfying the provisions of the GCT is a system of concurrent chemical reactions usually called a chemical network. It is not, however, immediately evident whether or not chemical constituents interacting through stimulus-response rules (chemical reactions) may form a network capable of solving intelligent tasks such as pattern recognition or computation. In this venue, the simplest model of a chemical neuron has been proposed by Okamoto et al. [10]. The possibility of connecting the Okamoto-type chemical neurons into a network has been analyzed in-depth in the series of publications by Hjelmfelt and Ross [11-14]. In particular, in Hjelmfelt et al. $[11,14]$ the feasibility of a chemical finite-state computing machine has been demonstrated; such a machine would include the most fundamental elements of traditional electronic computers, namely binary decoder, binary adder, stack of memory and internal clock. The possibility of a programmable chemical NN capable of storing patterns and solving pattern recognition problems has been proved in Hjelmfelt et al. [12]. At last, an ultimate computer science conjecture - whether or not a Turing Machine can be constructed from oscillating chemical reactions - has been also resolved affirmatively [13].

A systematic study of biochemical information-processing systems has been reported in [15]. A detailed comparison of computational capabilities of NNs and those of biochemical networks suggests the idea that these capabilities have very much in common. In a more general context, it should be noted that any system representable through NN may be considered as a version of a Turing Machine. And an even more powerful statement is also valid: any function computable by Turing Machine can be also computed by an appropriately parameterized processor net constructed of biochemical entities [16]. In practical terms, all this means that each biochemical network may be thought 
as an entity performing certain computation and may be formally represented through an appropriately constructed Turing Machine. And conversely, any function computable by a Turing Machine may also be computed by a specially designed biochemical network.

The famous question posed by Alan Turing in his groundbreaking paper "Can a machine think?" [17] continues to be a highly disputed topic in computer science, cognitive science and philosophy [18]. However, given the convincingly demonstrated equivalence between the NNs and Turing Machines, between the chemical networks and NNs, between NNs and population dynamics, etc., it seems reasonable to pose similar questions: "Can a chemical network think?"; "Can a population of dumb individuals, as a whole, think?"; "Can a microbial community think?; "Can a community of cells think?". From the discussion above, it is reasonable to infer that a swarm of locally interacting individuals lacking any personal intelligence can think at least in the same sense and at the same level of intelligence as Turing Machines and computers.

\section{Robotic communities}

A community of inanimate robots mutually interacting only through stimulus-response rules but lacking any analytical tools for premeditated collective strategy, is well qualified to be such a community of individuals interacting in accordance with LVS rules and satisfying the provisions of GCT. Proof of the principle that these communities may possess the elements of self-organization and swarm intelligence has been convincingly demonstrated in $[19,20]$. In these works, a group of memoryless micro-robots have been programmed to mimic individual behaviors of cockroaches. The micro-robots, however, were not hard-wired to have any analytical tools to gather information regarding behaviors of other robots or regarding the general plan of action. It has been shown experimentally that this community is capable of reproducing some patterns of collective behavior similar to those of real cockroaches. Division of labor in communities of robots has been studied in [21]. A comprehensive review of various aspects of swarm intelligence in communities of robots and biological entities is given in [22]. Cooperative behaviors in communities of autonomous mobile robots has been reviewed in [23].

Maltzahn et al. [24] constructed a system in which the synthetic biological and nanotechnological components communicate in vivo to enhance disease diagnostics and delivery of therapeutic agents. In these experiments, the swarms typically consisted of about one trillion nanoparticles. It has been shown "that communicating nanoparticle systems can be composed of multiple types of signaling and receiving modules, can transmit information through multiple molecular pathways, can operate autonomously and can target over 40 times higher doses of chemotherapeutics to tumors than non-communicating controls."

\section{Microbial communities}

Highly sophisticated forms of swarm intelligence have been observed in microbial communities. These communities represent a perfect example of species in competition governed by the LotkaVolterra dynamics [25-27]. Social organization of bacterial communities has been extensively analyzed in [28]. Bacterial communities are found to possess a form of inheritable collective memory and the ability of maintaining self-identity. Secondly, the bacterial communities are capable of collective decision-making, purposeful alterations of the colony structures, and recognition and identification of other colonies. In essence, bacterial communities as a whole may be seen as multicellular organisms with loosely organized cells and a sophisticated form of intelligence [29].

\section{Communities of somatic cells}

From the perspective of Lotka-Volterra dynamics, somatic cells are just another example of locally interacting units possessing, as a community, the emergent property of swarm intelligence. As mentioned in [29], "Bacteria invented the rules for cellular organization." However, in contrast to microbial communities which have a freedom of spatial restructuring, self-organization in a community of somatic cells is mostly manifested through collective shaping their internal phenotypic traits [30]. All this means that a community of somatic cells acts as a self-sufficient intelligent superorganism capable of taking care of its own survival through cooperative manipulation with intracellular states.

\section{Disruption of quorum sensing as a prerequisite for triggering carcinogenesis}

Carcinogenesis is a complex systemic phenomenon encompassing the entire hierarchy of biological organization. A great emphasis in carcinogenesis is placed on the role of disruption of the cell-to-cell signaling. With destruction of signaling pathways, not only the normal regulation of individual cellular processes is damaged, but also a blow is dealt to the, so to speak, mental capabilities of the community as a whole. Its collective memory is wiped out or distorted, customary division of labor between subpopulations is shifted towards aberrant modalities, community-wide self-defensive mechanisms are weakened or broken. In summary, the community as a whole falls into the state of disarray and amnesia in which it is feverishly searching for new ways towards survival. These processes in turn cause shift in expression profiles and metabolic dynamics and eventually penetrates to the level of DNA causing multiple mutations.

Quorum sensing (QS) is an important aspect of swarm intelligence. Agur et al. [31] provide a brief review of relevant biological facts and propose a mathematical model of QS boiled down to its simplest mechanistic elements. They arrive to important insight that "that cancer initiation is driven by disruption of the QS mechanism, with genetic mutations being only a side-effect of excessive proliferation." Detailed analysis of societal interactions and quorum sensing mechanisms in ovarian cancer metastases is given in [32]. These authors present compelling arguments supporting the view that QS "provides a unified and testable model for many long-observed behaviors of metastatic cells."

\section{Swarm intelligence is a key to understanding acquired chemoresistance}

Numerous observations confirm the notion that a cancer tumor may be regarded as a society of cells possessing the faculty of swarm intelligence. One of the important aspects of swarm intelligence is adaptivity which is a form of learning from experience.

It has been also long recognized that cancer cells, after the fleeting inhibitory effect of a chemotherapeutic agent, may develop the capabilities of resistance to treatment. These capabilities termed as acquired resistance, are the manifestations of robustness of cancer cells, both individually and collectively. In literature, in attempts to conceptualize this complex phenomenon, there is a reductionist tendency to associate adaptivity with multiple layers of negative feedback loops [33]. It is obvious, however, that the entire system 
comprising myriads of such loops cannot succeed in fulfilling its task unless these individual controls are working coherently, sharing a common goal. Observed astounding coherence between all the innumerable elementary processes comprising tumor adaptivity allows one to see tumor as a separate organ $[34,35]$ and to talk about its defensive tactics [36]. Fundamentally, such capabilities are nothing else than manifestations of swarm intelligence in the community of tumor cells. It is, therefore, admissible to hypothesize that, when developing therapeutic strategies against cancer, one needs to recognize that the enemy is intelligent, capable of discerning the weapon applied against it and mounting a counteroffensive.

\section{Conclusion}

Complex hierarchy of perfectly organized entities is a hallmark of biological systems. Attempts to understand why's and how's of this organization lead inquiring minds to various levels of abstraction and depths of interpretation. In this paper, we have attempted to convey the notion that there exists a set of comparatively simple and universal laws of nonlinear dynamics which shape the entire biological edifice as well as all of its compartments. These laws are equally applicable to individual cells, as well as to biochemical networks within the cells, as well as to the societies of cells, as well as to the societies other than the societies of cells, as well as to the populations of individual organisms. These laws are blind, automatic, and universal; they do not require existence of a supervisory authority, system-wide informational infrastructure or some sort of premeditated intelligent design. In large populations of individuals interacting only by stimulus-response rules, these laws generate a large variety of emergent phenomena with self-organization and swarm intelligence being their natural manifestations.

\section{References}

1. Garnier S, Gautrails J, Theraulas G (2007) The biological principles of swarm intelligence. Swarm Intelligence 1: 3-31.

2. Bose NK, Liang P (2012) Neural Networkk Fundamentals with Graphs, Algorithms, and Applications. New York, Tokyo, Toronto: McGraw-Hill, Inc.

3. Haykin S (1998) Neural Networks: A Comprehensive Foundation. Upper Saddle River, NJ: Prentice Hall.

4. Ikeda M, Siljak DD (1980) Lotka-Volterra Equations: Decomposition, Stability and Structure. J Math Biology 9: 65-83.

5. Grossberg S (1978a) Competition, Decision and Consensus. J Math Anal Appl 662: 470-493.

6. Grossberg S (1977) Pattern Formation by the Global Limits of a Nonlinear Competitive Interactions in n Dimensions. J Math Biol 4: 237-256. [Crossref]

7. Grossberg S (1978b) Decisions, patterns, and oscillations in nonlinear competitive systems with applications to Volterra-Lotka systems. J TheorBiol 73: 101-130. [Crossref]

8. Grossberg S (1980) Biological competition: decision rules, pattern formation, and oscillations. Proc Natl Acad Sci USA 77: 2338-2342. [Crossref]

9. Lotka AJ (1922) Natural selection as a physical principle. Proc Natl Acad Sci USA 8: 151-154. [Crossref]

10. Okamoto M, Sakai T, Hayashi K (1988) Biochemical switching device realizing McCulloch-Pitts type equation. Biol Cybern 58: 295-299. [Crossref]

11. Hjelmfelt A, Ross J(1992) Chemical implementation and thermodynamics of collective neural networks. Proc Natl Acad Sci USA 89: 388-391. [Crossref]

12. Hjelmfelt A, Schneider FW, Ross J (1993) Pattern recognition in coupled chemical kinetic systems. Science 260: 335-337. [Crossref]

13. Hjelmfelt A, Weinberger ED, Ross J (1991) Chemical implementation of neural networks and Turing machines. Proc Natl Acad Sci USA 88: 10983-10987. [Crossref]

14. Hjelmfelt A, Weinberger ED, Ross J (1992) Chemical implementation of finite-state machines. Proc Natl Acad Sci USA 891: 383-387. [Crossref]
15. Filo O, Lotan N (2010) Information Processing by Biochemical Systems. Hoboken, NJ: John Wiley and Son.

16. Siegelman H, Sontag E (1991) Turing Computability with Neural Nets. Applied Mathematics Letters 4: 77-80.

17. Turing A (1950) Computing Machinery and Intelligence. Mind 59: 433-460.

18. Penrose R (1994) Shadows of the Mind. A Search for the Missing Science of Consciousness. Oxford, New York, Melburne: Oxford University Press.

19. Garnier S, Jost C, Gautrais J, Asadpour M, Caprari G, et al. (2008) The embodiment of cockroach aggregation behavior in a group of micro-robots. Artif Life 14: 387-408. [Crossref]

20. Garnier S, Jost C, Jeanson R, Gay C, Asadpour M, et al. (2005) Aggregation behavior as a source of collective decision in a group of cockroach-like-robots. Advances in Artificial Life. Lecture Notes in Computer Science: 169-178.

21. Labella T, Dorigo M, Deneubourg JL (2006) Division of Labor in a Group of Robots Inspired by Ants' Foraging Behavior. ACM Transactions on Autonomous and Adaptive Systems 1: 4-25.

22. Bonabeau E, Dorigo M, TheraulazG (1999) Swarm Intelligence: From Natural to Artificial Systems. New York, Oxford: Oxford University Press.

23. Cao Y, Fukunaga A, Kangh A (1997) Cooperative Mobile Robotics: Antecedents and Directions. Cooperative Mobile Robotics 7-27.

24. Maltzahn G, Park JH, Lin K, Singh N, Schwoppe C, et al. (2011) Nanoparticles that communicate in vivo to amplify tumor targeting. Nat Mater 10: 545-552. [Crossref]

25. Gaki A, Theodorou A, Vayenas DV, Pavlou S (2009) Complex dynamics of microbial competition in the gradostat. J Biotechnol 139: 38-46. [Crossref]

26. Kooi BW, Boer MP, Kooijman SALM (1997) Complex Dynamic Behavior of Autonomous Microbial Food Chains. Journal of Mathematical Biology 36: 24-40.

27. Pepper JW, Rosenfeld S (2012)The emerging medical ecology of the human gut microbiome. Trends Ecol Evol 27: 381-384. [Crossref]

28. Ben-Jacob E, Becker I, Shapira Y, Levine H (2004) Bacterial linguistic communication and social intelligence. Trends Microbiol 12: 366-372. [Crossref]

29. Bassler BL (1999) How bacteria talk to each other: regulation of gene expression by quorum sensing. Curr Opin Microbiol 2: 582-587. [Crossref]

30. Seeley TD (2002) When is self-organization used in biological systems? Biol Bull 202: 314-318. [Crossref]

31. Agur Z, Kogan Y, Levi L, Harrison H, Lamb R, et al. (2010) Disruption of a Quorum Sensing mechanism triggers tumorigenesis: a simple discrete model corroborated by experiments in mammary cancer stem cells. Biol Direct 5: 20. [Crossref]

32. Hickson J, Diane YS, Berger J, Alverdy J, O'Keefe J, et al. (2009) Societal interactions in ovarian cancer metastasis: a quorum-sensing hypothesis. Clin Exp Metastasis 26: 67-76. [Crossref]

33. Kitano H (2004) Cancer as a robust system: implications for anticancer therapy. Nat Rev Cancer 4: 227-235. [Crossref]

34. Egeblad M, Nakasone ES, Werb Z (2010) Tumors as organs: complex tissues that interface with the entire organism. Dev Cell 18: 884-901. [Crossref]

35. Radisky D, Hagios C, Bissell MJ (2001) Tumors are unique organs defined by abnormal signaling and context. Semin Cancer Biol 11: 87-95. [Crossref]

36. Kitano H (2003) Cancer robustness: tumour tactics. Nature 426: 125. [Crossref]

Copyright: (C2015 Rosenfeld S. This is an open-access article distributed under the terms of the Creative Commons Attribution License, which permits unrestricted use, distribution, and reproduction in any medium, provided the original author and source are credited. 